\title{
Prognosis of Biliary Atresia Associated With Cytomegalovirus: A Meta-Analysis
}

\author{
Yilin Zhao ${ }^{1,2}$, Xiaodan Xu ${ }^{1,2}$, Gengxin Liu ${ }^{1,2}$, Fang Yang ${ }^{1,2}$ and Jianghua Zhan ${ }^{2 *}$ \\ ${ }^{1}$ Graduate College, Tianjin Medical University, Tianjin, China, ${ }^{2}$ Department of General Surgery, Tianjin Children's Hospital, \\ Tianjin, China
}

Objective: The etiology of biliary atresia is unclear, but viral infection has been implicated. The aim of the current meta-analysis was to investigate relationships between cytomegalovirus (CMV) and the prognosis of biliary atresia.

Methods: PubMed, Embase, the Cochrane Library, the China National Knowledge Infrastructure database, and Wanfang Data electronic databases were searched for eligible studies. Each relevant text was thoroughly reviewed and examined, including related papers in their reference lists.

OPEN ACCESS

Edited by:

Pietro Vajro

University of Salerno, Italy

Reviewed by:

Augusto Zani,

Hospital for Sick Children, Canada

Björn Fischler,

Karolinska University

Hospital, Sweden

Mark Davenport

Kings Health Partners,

United Kingdom

*Correspondence:

Jianghua Zhan

zhanjianghuatj@163.com

Specialty section: This article was submitted to

Pediatric Gastroenterology,

Hepatology and Nutrition,

a section of the journal

Frontiers in Pediatrics

Received: 16 May 2021

Accepted: 16 July 2021

Published: 18 August 2021

Citation:

Zhao Y, Xu X, Liu G, Yang F and

Zhan J (2021) Prognosis of Biliary

Atresia Associated With

Cytomegalovirus: A Meta-Analysis.

Front. Pediatr. 9:710450.

doi: 10.3389/fped.2021.710450
Results: A total of nine studies including 784 patients were included in the analysis. Biliary atresia patients with CMV exhibited significantly lower jaundice clearance (odds ratio: $0.46, p<0.0001 ; l^{2}=15 \%, p=0.31$ ). There were no significant differences in the rates of cholangitis or native liver survival. CMV-pp65-positive biliary atresia patients had a significantly lower rate of jaundice clearance (odds ratio: $5.87, p=0.003 ; l^{2}=0 \%, p$ $=0.71$ ) and a significantly higher rate of cholangitis (odds ratio: $0.21, p=0.01 ; l^{2}=0 \%$, $p=0.43$ ) than CMV antibody-positive biliary atresia patients.

Conclusion: Biliary atresia patients who were also infected with CMV had a poorer prognosis, particularly with respect to jaundice clearance. CMV status may influence the prognosis of biliary atresia. Clinicians should be able to routinely identify the subset of biliary atresia patients who are also CMV-positive, in order to improve native liver survival.

Keywords: biliary atresia, cytomegalovirus, jaundice clearance, meta-analysis, prognosis

\section{HIGHLIGHTS}

- The current systematic review and meta-analysis was performed to investigate relationships between cytomegalovirus (CMV) and the prognosis of biliary atresia (BA).

- BA patients with CMV had worse prognoses, particularly with respect to jaundice clearance.

- CMV-pp65-positive BA patients exhibited lower jaundice clearance and a higher incidence of cholangitis than CMV-Ig-positive BA patients.

- It is important for clinicians to detect CMV infection early via different methods in children with BA.

\section{INTRODUCTION}

Biliary atresia (BA) is a destructive and obliterative cholangiopathy that occurs in infants. It involves both the extra-hepatic and the intra-hepatic bile ducts, and it is characterized by progressive inflammation and fibrosis of the biliary tree (1). The incidence of BA ranges from 1/5,000 to $1 / 20,000$, and it is particularly high in Asian countries $(2,3)$. The main treatment for BA is currently 
Kasai portoenterostomy (KPE), which re-establishes bile flow and prolongs the survival of the native liver $(4,5)$. The etiology of BA may be associated with immune dysregulation, inflammation, and virus infection, but it is still unclear $(6,7)$. Cytomegalovirus (CMV) affects BA. Evidence of CMV infection has been reported in $10-38 \%$ of infants with BA in series from England, Germany, Brazil, and Sweden, but up to $60 \%$ in China $(4,6,8)$. In a series of studies, CMV-associated BA patients exhibited poor outcomes, but other studies have yielded inconsistent results. These inconsistences may be due to the relatively small sample sizes involved. The present study aimed to investigate outcomes in patients with CMV-associated BA after KPE.

\section{MATERIALS AND METHODS}

The present review and meta-analysis were conducted in accordance with the Assessing the Methodological Quality of
Systematic Reviews checklist and the Preferred Reporting Items for Systematic Reviews and Meta-Analyses guidelines.

\section{Search Strategy}

An extensive search was conducted for studies that investigated associations between CMV and BA. Data published up to December 2020 were sourced from the PubMed, Embase, Cochrane Library, China National Knowledge Infrastructure, and Wanfang Data electronic databases. All relevant reports were thoroughly reviewed, including those in reference lists. The search terms were (Biliary Atresia) OR (Atresia, Biliary) OR (Intrahepatic Biliary Atresia) OR (Atresia, Intrahepatic Biliary) OR (Biliary Atresia, Intrahepatic) OR (Biliary Atresia, Extrahepatic) OR (Atresia, Extrahepatic Biliary) OR (Extrahepatic Biliary Atresia) OR (Idiopathic Extrahepatic Biliary Atresia) OR (Familial Extrahepatic Biliary Atresia) OR (bile duct atresia) OR (biliary atresia) OR [ "Biliary Atresia" [Mesh]] AND [ "Cytomegalovirus Infections" [Mesh]] OR

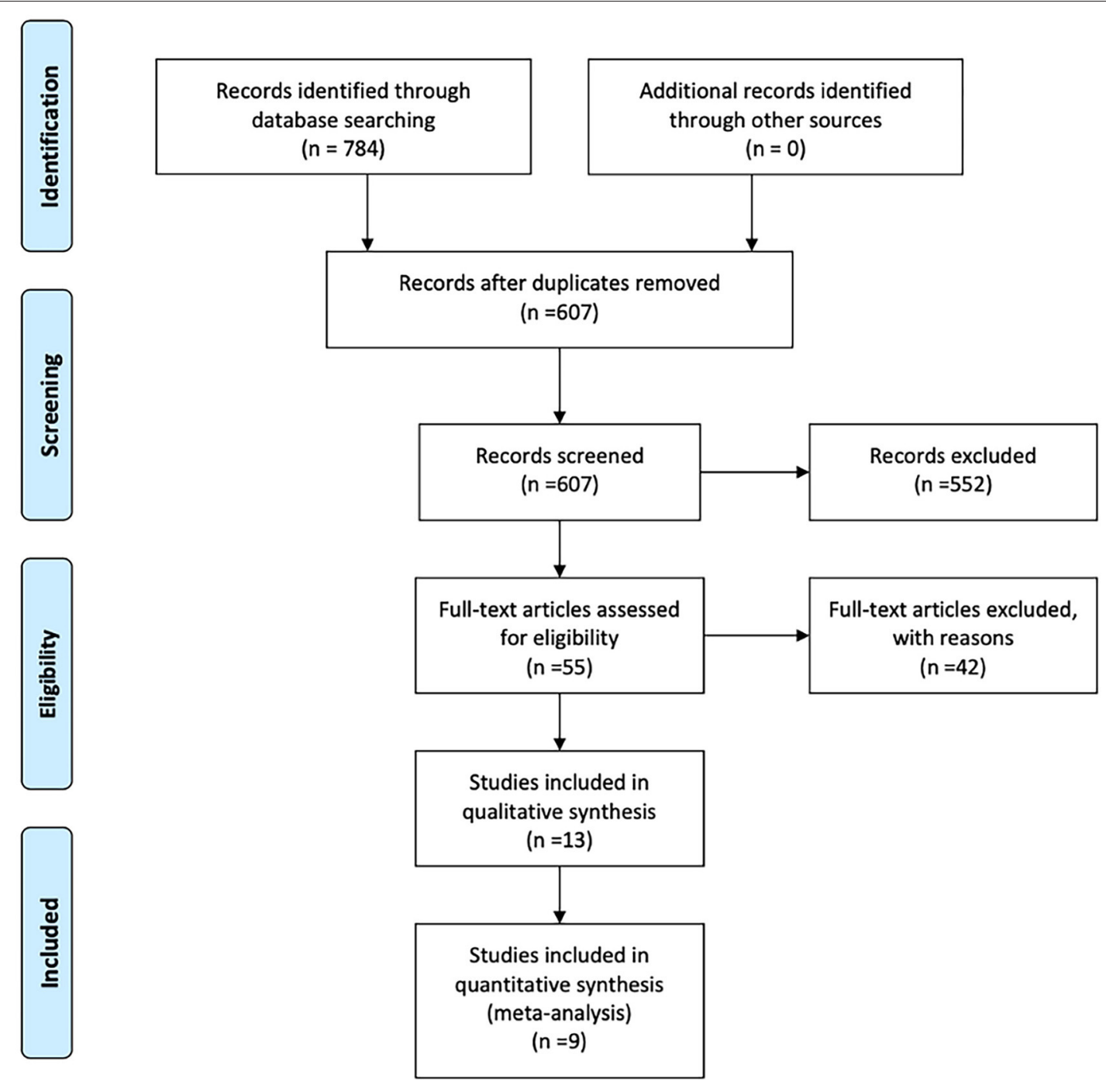

FIGURE 1 | Flowchart of the study selection process. 
["Cytomegalovirus" (Mesh)] OR (cytomegaloviruses) OR (Infections, Cytomegalovirus) OR (Cytomegalovirus Infection) OR (Infection, Cytomegalovirus) OR (Cytomegalic Inclusion Disease) OR (Cytomegalic Inclusion Diseases) OR (Disease, Cytomegalic Inclusion) OR (Diseases, Cytomegalic Inclusion) OR (Inclusion Disease, Cytomegalic) OR (Inclusion Diseases, Cytomegalic) OR (Cytomegalovirus Infections). The search was conducted without restrictions on language or year of publication.

\section{Study Selection and Data Extraction}

The inclusion criteria were (1) studies that included CMV detection in serum or urine; (2) randomized clinical trials (RCTs) or prospective/retrospective studies comparing $\mathrm{CMV}^{+}$ $\mathrm{BA}$ and $\mathrm{CMV}^{-} \mathrm{BA}$; (3) studies that included at least one of the following outcomes: "clearance of jaundice," "cholangitis," "NLS in 2 years," or "survival curve on NLS"; and (4) studies with scores $\geq 6$. Case reports, meeting abstracts, review articles, letters, and opinions were excluded. Two investigators independently assessed the eligibility of the studies by reviewing titles, abstracts, and, when required, full texts. Differences were settled via discussion. The extracted data recorded included first author, year of publication, study type, number of patients, jaundice clearance and cholangitis data, rate of native liver survival (NLS) in 2 years, and NLS survival curves. CMV can be detected via several methods. In the present analysis, $\mathrm{CMV}^{-} \mathrm{BA}$ was defined as all negative indicators in both blood samples and urine samples, and any deviation from that was defined as $\mathrm{CMV}^{+}$ BA. CMV-Ig-positive BA was defined as CMV antibody-positive but CMV-pp65-negative, and patients who were positive for CMV-pp65 were defined as CMV-pp65-positive.

\section{Statistical Analysis and Exploration of Heterogeneity}

Stata SE15.0 was used for the analysis. Odds ratios (ORs) with 95\% confidence intervals (CIs) were calculated based on the reported numbers of patients and events. Hazard ratios (HRs) with 95\% CIs were calculated according to calculate $\ln H \mathrm{R}$ and its variance by NLS survival curves (9). Mean differences (MDs) with $95 \%$ CIs were used for continuous outcomes. Differences of $p<0.05$ were considered statistically significant. Heterogeneity was assessed via the Cochrane Q test and $I^{2}$ values (10). $p<0.1$

TABLE 1 | Characteristics of the reports included in the meta-analysis.

\begin{tabular}{|c|c|c|c|c|c|c|c|c|c|c|c|c|}
\hline \multirow[t]{2}{*}{ Reference } & \multirow[t]{2}{*}{ Year } & \multirow[t]{2}{*}{$\begin{array}{l}\text { Study } \\
\text { type }\end{array}$} & \multicolumn{2}{|c|}{$\begin{array}{l}\text { Jaundice } \\
\text { clearance }\end{array}$} & \multicolumn{2}{|c|}{$\begin{array}{l}\text { Incidence of } \\
\text { cholangitis }\end{array}$} & \multicolumn{2}{|c|}{$\begin{array}{c}\text { 2-year native liver } \\
\text { survival }\end{array}$} & \multicolumn{2}{|c|}{$\mathrm{CMV}^{+}$sample } & \multirow[t]{2}{*}{$\begin{array}{l}\mathrm{CMV}^{-} \\
\text {sample }\end{array}$} & \multirow[t]{2}{*}{$\begin{array}{l}\text { NOS } \\
\text { score }\end{array}$} \\
\hline & & & $\exp$ & control & $\exp$ & control & $\exp$ & control & CMV-Ig ${ }^{+}$ & CMV-pp65 ${ }^{+}$ & & \\
\hline Zani et al. (6) & 2015 & Pro & $3 / 20$ & $57 / 109$ & - & - & - & - & & 20 & 109 & 7 \\
\hline Chun et al. (12) & 2008 & $\operatorname{Re}$ & $13 / 22$ & $4 / 5$ & $6 / 22$ & $1 / 5$ & - & - & 11 & 11 & 5 & 6 \\
\hline Fischler et al. (11) & 2008 & $\mathrm{Re}$ & - & - & - & - & - & - & & 11 & 17 & 7 \\
\hline Song et al. (13) & 2017 & $\operatorname{Re}$ & $22 / 35$ & $80 / 123$ & - & - & 20/35 & $69 / 123$ & & 35 & 123 & 6 \\
\hline Ji (16) & 2020 & $\mathrm{Re}$ & $48 / 76$ & $13 / 20$ & - & - & - & - & & 76 & 20 & 6 \\
\hline Lv et al. (15) & 2019 & $\operatorname{Re}$ & $8 / 21$ & 26/39 & - & - & - & - & & 21 & 39 & 6 \\
\hline Qu et al. (17) & 2013 & $\operatorname{Re}$ & 19/39 & $17 / 23$ & - & - & 17/39 & $16 / 23$ & & 39 & 23 & 6 \\
\hline Luo et al. (18) & 2007 & $\operatorname{Re}$ & $24 / 38$ & $8 / 9$ & $13 / 38$ & $1 / 9$ & - & - & 20 & 18 & 9 & 6 \\
\hline Dong et al. (14) & 2016 & $\mathrm{Re}$ & 20/32 & $43 / 55$ & - & - & - & - & & 32 & 55 & 6 \\
\hline Total & - & - & - & - & - & - & - & - & & 294 & 400 & - \\
\hline
\end{tabular}

CMV, cytomegalovirus; exp, experimental; NOS, Newcastle-Ottawa Scale; Pro, prospective; Re, retrospective.

TABLE 2 | Data from the reports included in the meta-analysis.

\begin{tabular}{|c|c|c|c|c|}
\hline References & Detection of CMV-related BA & $\begin{array}{l}\text { Jaundice clearance } \\
\text { standards }\end{array}$ & $\begin{array}{l}\text { Cholangitis } \\
\text { standards }\end{array}$ & Treatment \\
\hline Zani et al. (6) & CMV IgM in serum & Bilirubin $\leq 20 \mu \mathrm{mol} / \mathrm{L}$ in $6 \mathrm{~m}$ & - & No antiviral drugs \\
\hline Chun et al. (12) & One of CMV IgM, IgG, or pp65 in serum & $\mathrm{TB}<34 \mu \mathrm{mol} / \mathrm{L}$ in $3 \mathrm{~m}$ & Within $6 \mathrm{~m}$ & - \\
\hline Fischler et al. (11) & CMV IgM in serum or urine & - & - & No antiviral drugs \\
\hline Song et al. (13) & Antibody in serum & Inexact definition in $3 \mathrm{~m}$ & - & - \\
\hline $\mathrm{Ji}(16)$ & IgM in serum & Bilirubin $<20 \mu \mathrm{mol} / \mathrm{L}$ in $2 \mathrm{y}$ & - & - \\
\hline Lv et al. (15) & CMV DNA in serum > 500 copies $/ \mathrm{mL}$ & $\mathrm{TB}<34.2 \mu \mathrm{mol} / \mathrm{L}$ in $6 \mathrm{~m}$ & - & - \\
\hline Qu et al. (17) & CMV antibody in serum & Bilirubin $<20 \mu \mathrm{mol} / \mathrm{L}$ in $6 \mathrm{~m}$ & - & - \\
\hline Luo et al. (18) & One of lgM, lgG, or pp65 in serum & $\begin{array}{l}\mathrm{TB}<34 \mu \mathrm{mol} / \mathrm{L} \text { and } \mathrm{DB}<17 \\
\mu \mathrm{mol} / \mathrm{L} \text { in } 3 \mathrm{~m}\end{array}$ & Within $6 \mathrm{~m}$ & - \\
\hline Dong et al. (14) & $\lg M$ & In $6 \mathrm{~m}$ & - & No antiviral drugs \\
\hline
\end{tabular}

BA, biliary atresia; CMV, cytomegalovirus; $D B$, direct bilirubin; $m$, months; TB, total bilirubin; $y$, years. 
or $I^{2}<50 \%$ was considered to indicate low heterogeneity and then a fixed effects model was used; otherwise, a random effects model was used (10).

\section{Quality Assessment and Publication Bias}

The quality of the studies included was evaluated via the Newcastle-Ottawa Scale (NOS) by two reviewers independently. Studies with NOS scores $\geq 6$ were considered high quality and were included in analyses. Publication bias and jaundice clearance results were evaluated via Egger's test, but other parameters were not analyzed due to the small number of studies.

\section{RESULTS}

\section{Search Results and Study Characteristics}

A flowchart of the search results is shown in Figure 1. A total of 784 records were identified and evaluated by two investigators

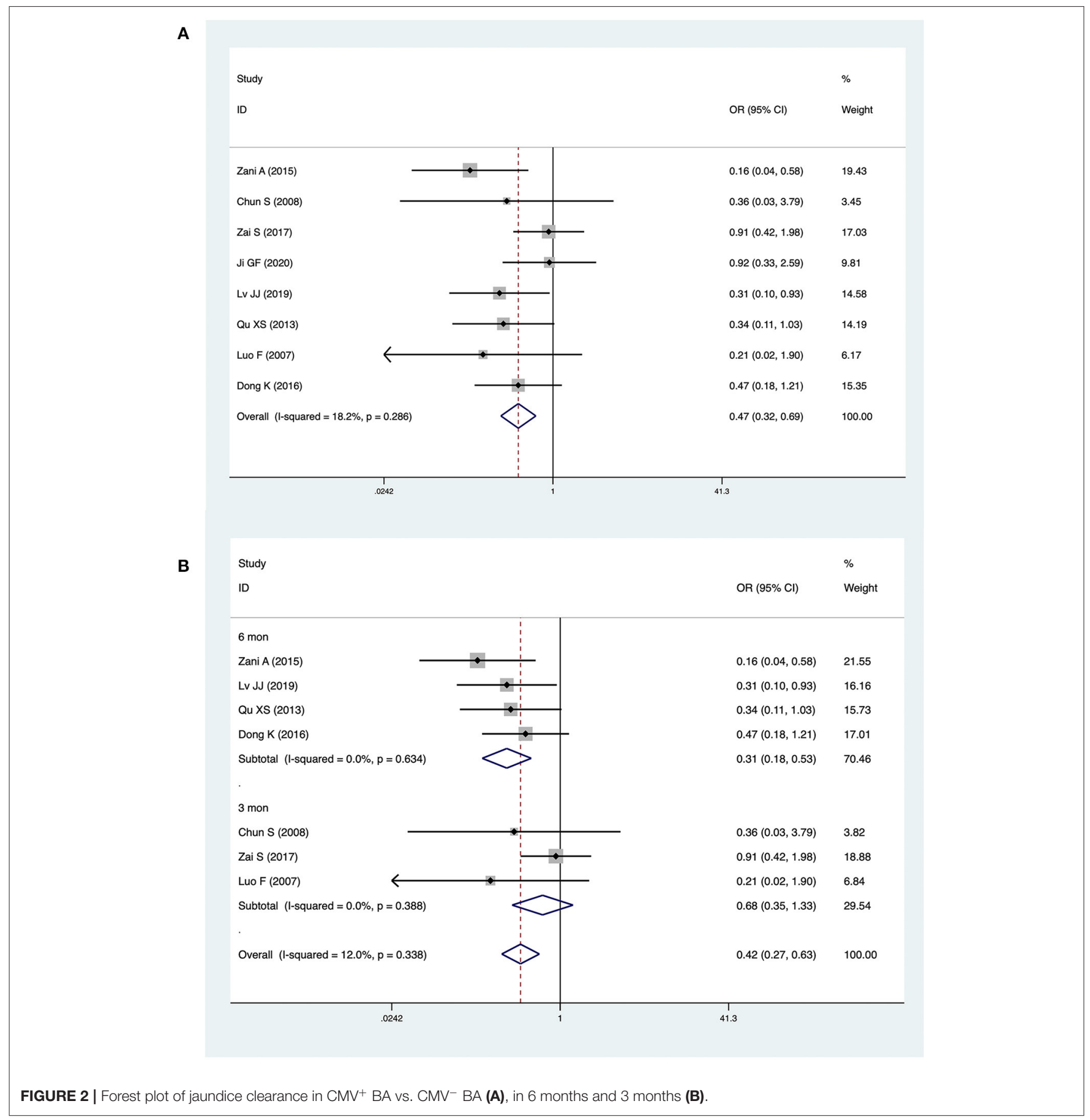


independently. After excluding 177 that were duplicates, a further 552 were excluded after screening the titles and abstracts. A further 42 studies were excluded due to no access to the full text or complete data. Nine studies published between 2007 and 2020 ultimately met the inclusion criteria and were included in the meta-analysis $(6,11-18)$. Detailed characteristics of the selected studies are summarized in Table 1. Eight of the studies included were retrospective and one was prospective, and collectively they included a total of 694 BA patients. Of these, 294 were classified as $\mathrm{CMV}^{+}$BA patients. Characteristics of the reports included are shown in Tables 1, 2.

\section{Jaundice Clearance}

Eight studies with a total of 666 patients $\left(283 \mathrm{CMV}^{+} \mathrm{BA}\right.$, $383 \mathrm{CMV}^{-}$) described jaundice clearance. The pooled OR was 0.47 (95\% CI: $0.32-0.69, p<0.001$ ) (Figure 2A). Heterogeneity among the studies was low $\left(I^{2}=18.2 \%, p=0.286\right)$. Three studies reported jaundice clearance within 6 months. The pooled OR was 0.31 (95\% CI: $0.18-0.53, p<0.001)$, and heterogeneity was not significant $\left(I^{2}=0.0 \%, p=0.634\right)$ (Figure 2B). Three studies reported jaundice clearance within 3 months (pooled OR: 0.68, 95\% CI: 0.35-1.33, $p=0.256, I^{2}=0.0 \%$ ) (Figure 2B).

\section{Incidence of Cholangitis}

Two studies including 74 patients reported cholangitis data. The pooled OR was 2.76 (95\% CI: 0.57-13.45, $p=0.21$ ) and heterogeneity was not significant $\left(I^{2}=0.0 \%, p=0.534\right)$ (Figure 3). There was no significant difference in cholangitis incidence between $\mathrm{CMV}^{+} \mathrm{BA}$ and $\mathrm{CMV}^{-}$BA patients.

\section{NLS}

Four studies including 340 patients reported NLS survival curves. The pooled HR was 1.59 (95\% CI: 0.66-3.81, $p=0.301$ ), and heterogeneity was significant $\left(I^{2}=71.2 \%, p=0.015\right)$ (Figure 4A). In analyses restricted to studies conducted in China, there was no significant difference in NLS ( $\mathrm{HR}=1.24,95 \% \mathrm{CI}$ : $0.37-4.17, p=0.729)$ and heterogeneity was high $\left(I^{2}=60.4 \%, p\right.$ $=0.112)$ (Figure 4B).

\section{CMV-Ig and CMV-pp65 BA}

Two studies investigated differences between CMV-Ig-positive BA and CMV-pp65-positive BA. The pooled OR of jaundice clearance was 5.87 (95\% CI: 1.85-18.65, $p=0.003)$, and heterogeneity was low $\left(I^{2}=0.0 \%, p=0.714\right)$ (Figure 5A). The OR of cholangitis was 0.21 (95\% CI: $0.06-0.69, p=$ $0.010)$, and heterogeneity was not significant $\left(I^{2}=0.0 \%, p\right.$ $=0.426$ ) (Figure 5B). In Zai et al. (13), CMV-pp65-positive BA patients exhibited significantly lower jaundice clearance and a significantly higher incidence of cholangitis than CMV-Igpositive BA patients. Luo et al. (18) reported similar results.

\section{Others}

Four studies reported $\mathrm{KPE}$ times in $\mathrm{CMV}^{+} \mathrm{BA}$ and $\mathrm{CMV}^{-} \mathrm{BA}$ patients. The pooled result was 1.84 (95\% CI: -12.13 to $15.82, p=$ $0.796)$ and there was high heterogeneity $\left(I^{2}=80.3 \%, p=0.002\right)$ (Figure 6). There was no significant difference between $\mathrm{CMV}^{+}$ $\mathrm{BA}$ patients and $\mathrm{CMV}^{-}$BA patients. In Zani et al. (6), infants with $\mathrm{CMV}^{+} \mathrm{BA}$ were significantly older at the time of KPE. In Dong et al. (14) and Fischler et al. (11), patients with $\mathrm{CMV}^{+} \mathrm{BA}$ were older than those with $\mathrm{CMV}^{-} \mathrm{BA}$ at the time of KPE, but not statistically significantly. In Ji et al. (16), patients with $\mathrm{CMV}^{+} \mathrm{BA}$ were younger than those with $\mathrm{CMV}^{-} \mathrm{BA}$ at the time of $\mathrm{KPE}$, but not statistically significantly.

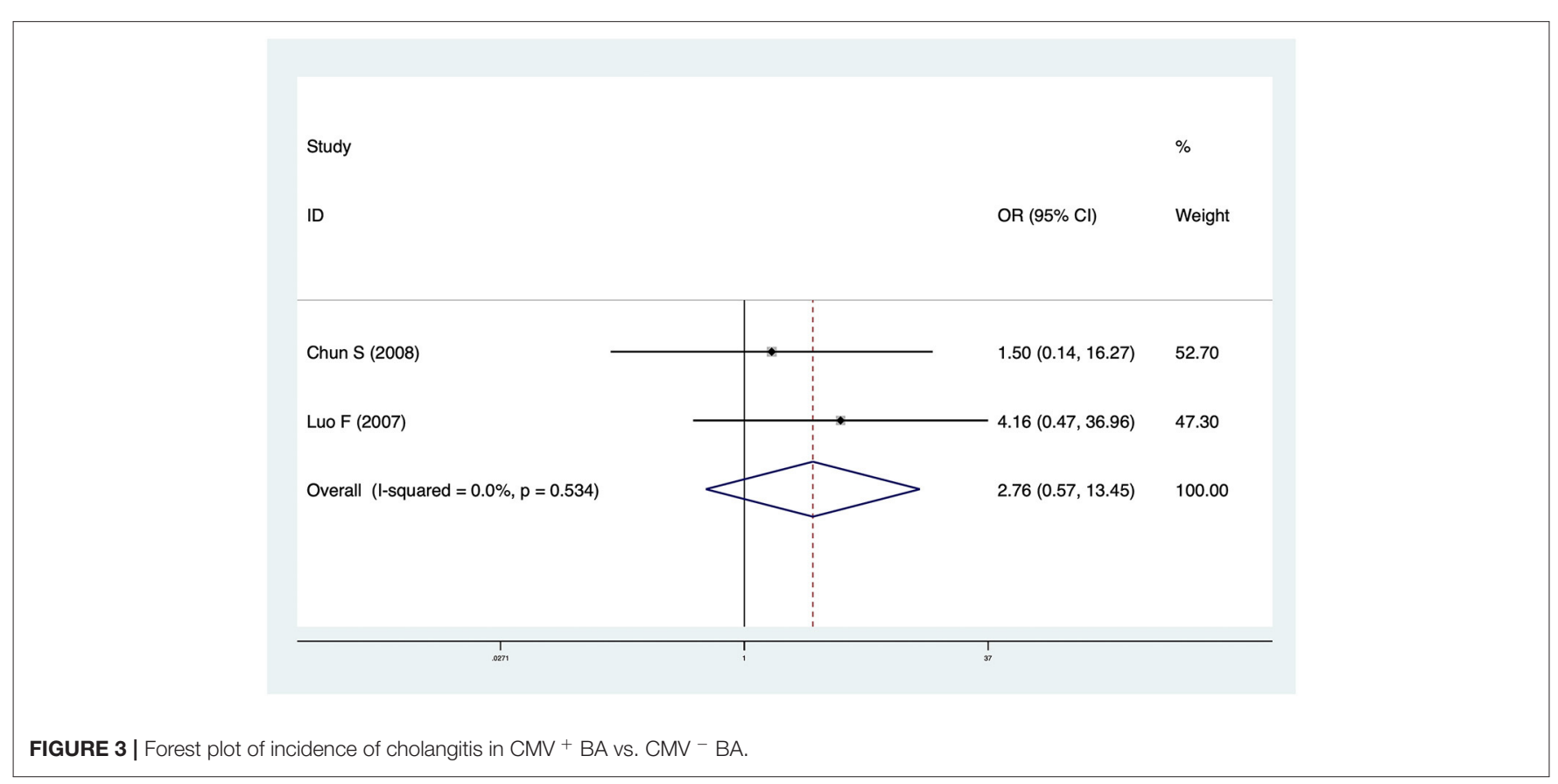


A

Study

$\%$

ID

HR $(95 \% \mathrm{Cl}) \quad$ Weight

Zani A (2015)

Fischler B (2008)

Ji GF (2020)

Dong K (2016)

Overall $($ I-squared $=71.2 \%, p=0.015)$
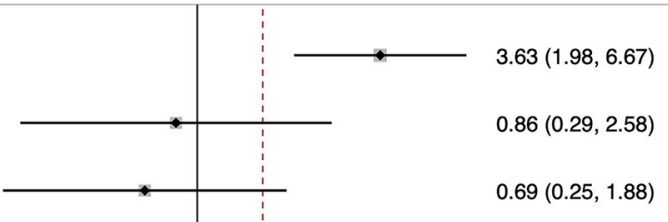

30.59

(1)

NOTE: Wegins are trom rantom entects anarassis

${ }_{.132}^{2}$

$\left.\right|_{1} ^{132}$

$0.86(0.29,2.58) \quad 22.93$

$0.69(0.25,1.88) \quad 24.43$

$2.39(0.75,7.59) \quad 22.05$

$1.59(0.66,3.81) \quad 100.00$

B

Study

$\%$

ID

$\mathrm{HR}(95 \% \mathrm{Cl}) \quad$ Weight

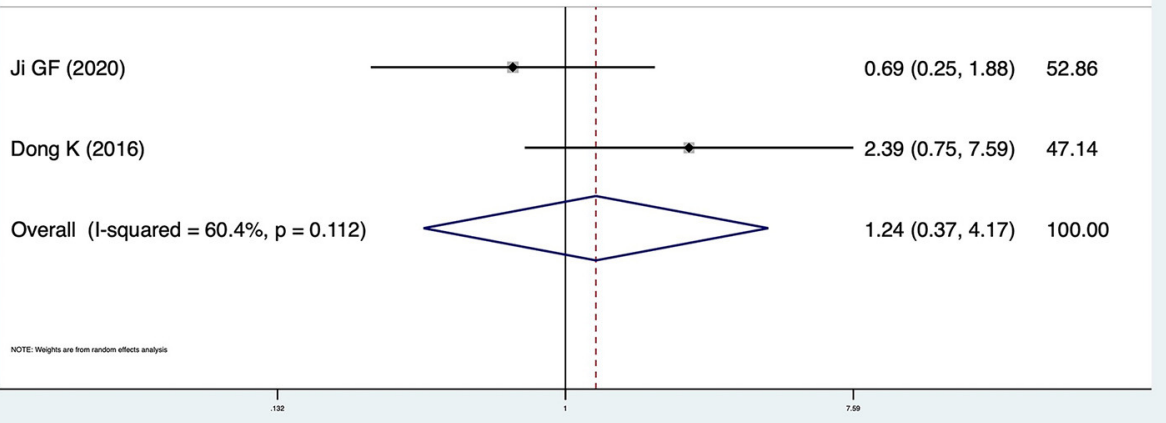

FIGURE 4 | (A) Forest plot of the NLS in CMV + BA vs. CMV - BA. (B) Forest plot of the NLS in CMV + BA vs. CMV - BA in China.

\section{Quality Assessment and Publication Bias}

NOS scores are shown in Table 1. An Egger's funnel plot derived from the studies describing jaundice clearance in the current meta-analysis is shown in Figure 7. Egger's test indicated no significant publication bias $(t=-1.77, p=0.127)$.

\section{DISCUSSION}

CMV is a hepatophilic double-stranded DNA virus (6). Domiati-Saad et al. (19) reported that CMV was related to BA in a PCR-based study conducted in the 1990's, and Fischler et al. (20) detected CMV-IgM in serum in BA patients in the same era. In 2012, Davenport (3) identified CMV-IgM biliary atresia as a new subgroup. In a series of studies, CMV affecting the prognosis of BA was not limited to CMV-IgM positivity. Accordingly, in the present study, any positive indicator in blood or urine was used to define $\mathrm{CMV}^{+} \mathrm{BA}$, and a lack of any such indicators was used to define $\mathrm{CMV}^{-}$BA. $\mathrm{CMV}^{+}$ $\mathrm{BA}$ is associated with a poor prognosis. In the present metaanalysis, patients with $\mathrm{CMV}^{+} \mathrm{BA}$ had a significantly lower rate of jaundice clearance. Some studies reported that $\mathrm{CMV}^{+} \mathrm{BA}$ patients presented symptoms later and were operated on later $(6,11,16) . \mathrm{CMV}^{+} \mathrm{BA}$ was associated with more severe fibrosis and inflammation during surgery than $\mathrm{CMV}^{-} \mathrm{BA}(12,14)$. There were indications that the virus triggered proinflammatory mechanisms, and this may lead to an autoimmune response guided by Th1 cells (21). At the time of KPE, liver fibrosis and inflammation were more severe in $\mathrm{CMV}^{+} \mathrm{BA}$ patients than in 


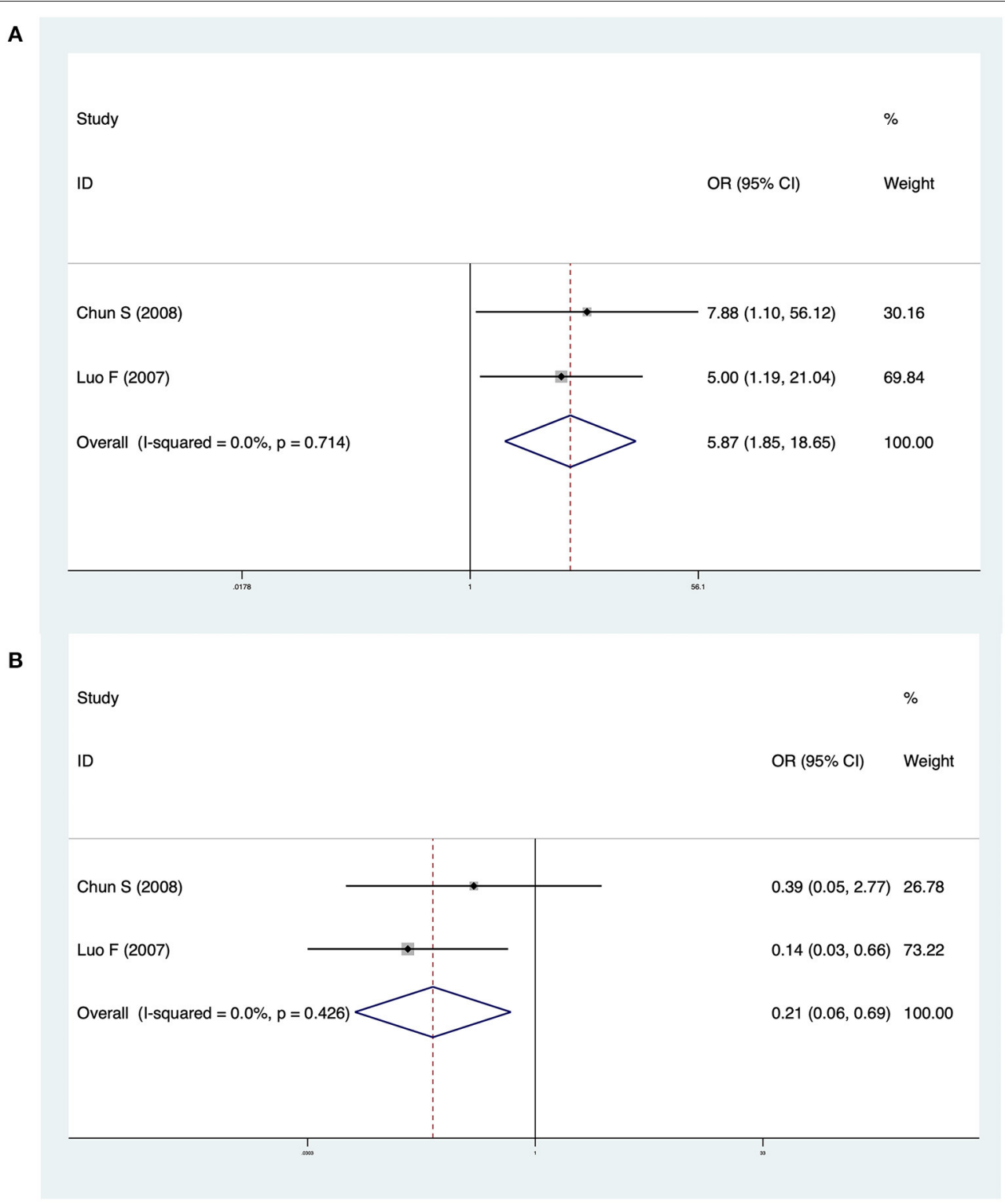

FIGURE 5 | (A) Forest plot of jaundice clearance in CMV-lg-positive BA vs. CMV-pp65-positive BA. (B) Forest plot of cholangitis in CMV-lg-positive BA vs. CMV-pp65-positive BA

$\mathrm{CMV}^{-} \mathrm{BA}$ patients. There was a higher rate of cholangitis in $\mathrm{CMV}^{+} \mathrm{BA}$ patients, but not statistically significantly. Cholangitis is one of the major complications of $\mathrm{BA}$, and it is associated with a poor KPE prognosis. Repeated cholangitis can promote fibrosis, obstruct bile flow, and exacerbate jaundice and cirrhosis $(22,23)$. There was no significant difference in the rate of cholangitis in this study. The limited number of studies and the small sample sizes involved in the current investigation may have affected the results of the analysis. Different centers may also have different adjuvant therapies and different surgical operation levels, and this may also have affected the results of the analysis.
Various test measurements were used to detect the presence and status of CMV, including serum CMV-IgG, CMV-IgM, and CMV-DNA. CMV-pp65 can be detected in serum and urine. Positive CMV-IgM indicates recent infection that has been cured. CMV-IgG can be transferred through the mother's placenta and then it disappears gradually over the subsequent 2 years. CMV-pp65 antigenemia indicates duplication of CMV. CMVDNA is an indicator of early active infection. In the present analysis, CMV-pp65-positive BA was associated with a poor outcome with respect to jaundice clearance and cholangitis. In Chun et al. (12) and Luo et al. (18), CMV-pp65-positive BA was associated with more rapid development of liver fibrosis 


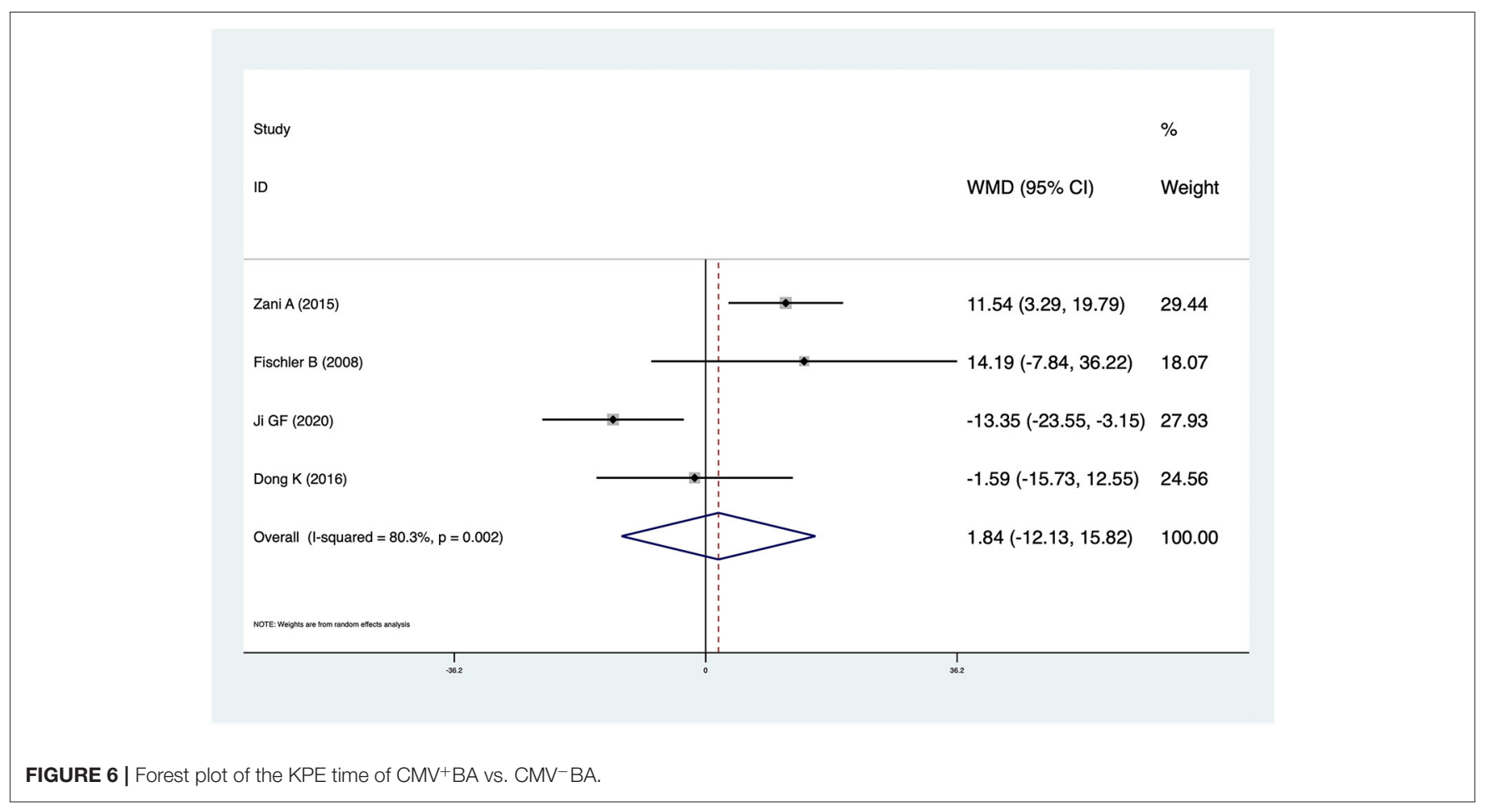

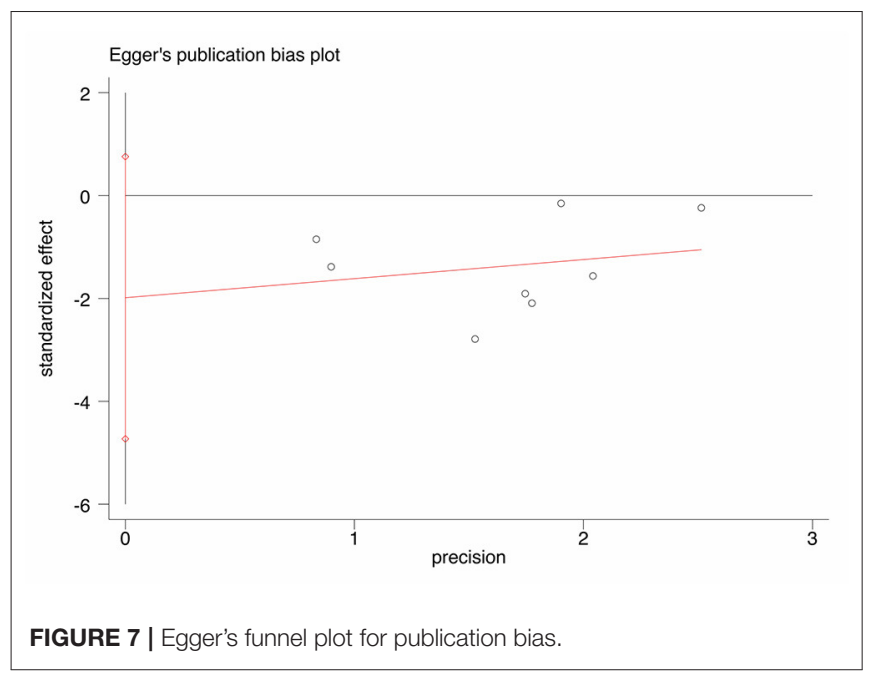

than in other BA patients, including those who were CMV antibody-positive. CMV status may influence the outcome of BA. It is essential for clinicians to test for CMV status. Measurements derived from serum or urine do not represent the status of the liver. It remains a challenge to diagnose active $\mathrm{CMV}$ infection in the liver. Most pediatric surgeons agree that CMV is one of the etiologies of BA. CMV inclusion bodies have not been detected in the liver in any reported studies. Many problems remain to be solved with respect to CMV in BA.
Antiviral therapy has been proposed to improve the prognosis of $\mathrm{CMV}^{+}$BA. In a study reported by Parolini et al. (24), antiviral drugs could negate the pathogenic effects of CMV, improve the rate of jaundice clearance, and reduce cholangitis. Notably however, there is not enough evidence to support the principle of adjuvant therapy, which currently depends on clinicians.

The present meta-analysis had several limitations. One was the lack of RCTs. Another was that the sample sizes of several of the studies included were relatively small. Some prognostic indicators were only included in a few of the studies analyzed, resulting in reduced statistical power and publication bias. No antiviral treatment was explicitly mentioned in any of the nine articles included. Lastly, there was a lack of comparisons of outcomes in $\mathrm{CMV}^{+} \mathrm{BA}$ patients undergoing antiviral therapy vs. $\mathrm{CMV}^{-}$BA patients.

In conclusion, the current meta-analysis indicates that CMV-IgM BA is a noteworthy subgroup of BA patients. BA patients with CMV exhibited poor prognosis, particularly with respect to jaundice clearance. CMV status may influence the prognosis of BA. Clinicians should be able to routinely identify $\mathrm{CMV}^{+} \mathrm{BA}$ patients, to facilitate attempts to improve NLS.

\section{DATA AVAILABILITY STATEMENT}

The original contributions presented in the study are included in the article/supplementary material, further inquiries can be directed to the corresponding author/s. 


\section{AUTHOR CONTRIBUTIONS}

YZ and FY contributed to the design of the research and perform statistics. YZ and XX contributed to design the statistical methods, search and choose studies, extracted the data, and pooled the results. YZ and GL interpreted the data, wrote and submitted the manuscript. JZ contributed to supervise the findings of this work, discussed the results, read and approved

\section{REFERENCES}

1. Asai A, Miethke A, Bezerra JA. Pathogenesis of biliary atresia: defining biology to understand clinical phenotypes. Nat Rev Gastroenterol Hepatol. (2015) 12:342-52. doi: 10.1038/nrgastro.2015.74

2. Chiu CY, Chen PH, Chan CF, Chang MH, Wu TC. Biliary atresia in preterm infants in Taiwan: a nationwide survey. J Pediatr. (2013) 163:1003.e101. doi: 10.1016/j.jpeds.2012.12.085

3. Davenport M. Biliary atresia: clinical aspects. Semin Pediatr Surg. (2012) 21:175-84. doi: 10.1053/j.sempedsurg.2012.05.010

4. Lakshminarayanan B, Davenport M. Biliary atresia: a comprehensive review. J Autoimmun. (2016) 73:1-9. doi: 10.1016/j.jaut.2016.06.005

5. Murase N, Hinoki A, Shirota C, Tomita H, Shimojima N, Sasaki H, et al. Multicenter, retrospective, comparative study of laparoscopic and open Kasai portoenterostomy in children with biliary atresia from Japanese high-volume centers. J Hepatobiliary Pancreat Sci. (2019) 26:43-50. doi: 10.1002/jhbp.594

6. Zani A, Quaglia A, Hadzić N, Zuckerman M, Davenport M. Cytomegalovirusassociated biliary atresia: an aetiological and prognostic subgroup. J Pediatr Surg. (2015) 50:1739-45. doi: 10.1016/j.jpedsurg.2015.03.001

7. Ortiz-Perez A, Donnelly B, Temple H, Tiao G, Bansal R, Mohanty SK. Innate immunity and pathogenesis of biliary atresia. Front Immunol. (2020) 11:329. doi: 10.3389/fimmu.2020.00329

8. Xu Y, Yu J, Zhang R, Yin Y, Ye J, Tan L, et al. The perinatal infection of cytomegalovirus is an important etiology for biliary atresia in China. Clin Pediatr. (2012) 51:109-13. doi: 10.1177/0009922811406264

9. Williamson PR, Smith CT, Hutton JL, Marson AG. Aggregate data meta-analysis with time-to-event outcomes. Stat Med. (2002) 21:333751. doi: 10.1002/sim.1303

10. Higgins JP, Thompson SG. Quantifying heterogeneity in a meta-analysis. Stat Med. (2002) 21:1539-58. doi: 10.1002/sim.1186

11. Fischler B, Svensson JF, Nemeth A. Early cytomegalovirus infection and the long-term outcome of biliary atresia. Acta Paediatr. (2009) 98:16002. doi: 10.1111/j.1651-2227.2009.01416.x

12. Shen C, Zheng S, Wang W, Xiao XM. Relationship between prognosis of biliary atresia and infection of cytomegalovirus. World J Pediatr. (2008) 4:123-6. doi: 10.1007/s12519-008-0024-8

13. Song Z, Dong R, Shen Z, Chen G, Yang Y, Zheng S. Surgical outcome and etiologic heterogeneity of infants with biliary atresia who received Kasai operation less than 60 days after birth: a retrospective study. Medicine. (2017) 96:e7267. doi: 10.1097/MD.0000000000007267

14. Dong K, Yang TQ, Dong CQ, Liu Q, Chen C, Su C, et al. Relationship between cytomegalovirus infection and prognosis of biliary atresia after Kasai operation. J Clin Pediatr Surg. (2016) 15:23-25+45. doi: 10.3969/j.issn.1671-6353.2016.01.007

15. Lv JJ, Wang Z, Zhong W. The influence of CMV infection on the prognosis of biliary atresia patients after Kasai's portoenterostomy. J Trop Med. (2019) 19:1024-7.

16. Ji GF. Analysis of prognostic factors in Kasai operation for biliary atresia (Dissertation). Shenyang: China Medical University (2020). the final manuscript. All authors approved the final version to be published.

\section{FUNDING}

This study was funded by the Tianjin Health Bureau special grant (Grant No: 14KG129) and the Tianjin Children's Hospital special grant (Grant No: Y2020002).

17. Qu XS, Zhang WQ, Li YC. Analysis of the short-and mid-term curative effect and influence factors of hepaticojejunostomy for biliary atresia. Chin J Prim Med Pharm. (2013) 20:2755-7. doi: 10.3760/cma.j.issn.1008-6706.2013. 18.014

18. Luo F, Wu XJ, Zhou DK. The Relationship between the prognosis of biliary atresia and the perinatal infection of cytomegalovirus. LASER J. (2007) 28:957. doi: 10.3969/j.issn.0253-2743.2007.05.051

19. Domiati-Saad R, Dawson DB, Margraf LR, Finegold MJ, Weinberg AG, Rogers BB. Cytomegalovirus and human herpesvirus 6, but not human papillomavirus, are present in neonatal giant cell hepatitis and extrahepatic biliary atresia. Pediatr Dev Pathol. (2000) 3:367-73. doi: 10.1007/s100240010045

20. Fischler B, Ehrnst A, Forsgren M, Orvell C, Nemeth A. The viral association of neonatal cholestasis in Sweden: a possible link between cytomegalovirus infection and extrahepatic biliary atresia. J Pediatr Gastroenterol Nutr. (1998) 27:57-64. doi: 10.1097/00005176-199807000-00010

21. Hill R, Quaglia A, Hussain M, Hadzic N, Mieli-Vergani G, Vergani $\mathrm{D}$, et al. Th-17 cells infiltrate the liver in human biliary atresia and are related to surgical outcome. J Pediatr Surg. (2015) 50:1297303. doi: 10.1016/j.jpedsurg.2015.02.005

22. Moore SW, Zabiegaj-Zwick C, Nel E. Problems related to CMV infection and biliary atresia. S Afr Med J. (2012) 102:890-2. doi: 10.7196/SAMJ.6163

23. Wu ET, Chen HL, Ni YH, Lee PI, Hsu HY, Lai HS, et al. Bacterial cholangitis in patients with biliary atresia: impact on short-term outcome. Pediatr Surg Int. (2001) 17:390-5. doi: 10.1007/s00383000 0573

24. Parolini F, Hadzic N, Davenport M. Adjuvant therapy of cytomegalovirus $\operatorname{IgM}+$ ve associated biliary atresia: prima facie evidence of effect. J Pediatr Surg. (2019) 54:1941-5. doi: 10.1016/j.jpedsurg.2018. 12.014

Conflict of Interest: The authors declare that the research was conducted in the absence of any commercial or financial relationships that could be construed as a potential conflict of interest.

Publisher's Note: All claims expressed in this article are solely those of the authors and do not necessarily represent those of their affiliated organizations, or those of the publisher, the editors and the reviewers. Any product that may be evaluated in this article, or claim that may be made by its manufacturer, is not guaranteed or endorsed by the publisher.

Copyright (C) 2021 Zhao, Xu, Liu, Yang and Zhan. This is an open-access article distributed under the terms of the Creative Commons Attribution License (CC BY). The use, distribution or reproduction in other forums is permitted, provided the original author(s) and the copyright owner(s) are credited and that the original publication in this journal is cited, in accordance with accepted academic practice. No use, distribution or reproduction is permitted which does not comply with these terms. 